\title{
Successful culture of Streptococcus pneumoniae from sputum despite dual anti-pneumococcal therapy using a novel biofilm disruption technique
}

\author{
Thomas A Cumbo ${ }^{1}$, Destin J Radder ${ }^{2}$, Saloni Patel ${ }^{3}$, Courtney Jarka ${ }^{3}$ and Christopher L Hutson ${ }^{4}$ \\ 'Infectious Diseases Division and CMO, Mount St. Mary's Hospital, Catholic Health of Buffalo, USA \\ ${ }^{2}$ BioSherpa LLC., USA \\ ${ }^{3}$ Department of Clinical Pharmacology, Catholic Health of Buffalo, USA \\ ${ }^{4}$ Department of Anaesthesia, CHI/St. Luke's Methodist Hospital, USA
}

\begin{abstract}
Sputum cultures often have low diagnostic yield. Overgrowth by commensal oral flora, environmental stresses, antibiotic use and clustering of pathogens in the sputum matrix can inhibit success. Moreover, pathogens are frequently protected in a native sputum biofilm. We have successfully cultured Streptococcus pneumoniae from a patient demonstrating signs and symptoms of active pneumococcal pneumonia using a novel biofilm disruption technique while on effective antibiotic therapy and despite the above stressors.
\end{abstract}

\section{Introduction}

Handling of respiratory specimens has evolved considerably. What was once a collection of sputum at the bedside with immediate application to growth media has become a several hours to several day delay in transport to a centralized laboratory in unstable environmental conditions. Prior studies demonstrated significant loss of pathogen viability as well as overgrowth of oral commensal bacteria in specimens that were not promptly processed $[1,2]$. This is especially notable for common pathogens such as S. pneumoniae and H. influenzae [3]. Delays of only three or four hours facilitate commensal bacterial overgrowth [4]. Unfortunately, modern molecular techniques are often difficult to interpret in polymicrobial samples and previous attempts to design transport media for expectorated sputum have failed [5]. Despite these shortcomings, guidelines continue to recommend sputum culture in all inpatients being empirically treated for potential multidrug-resistant organisms [6].

Natural tissue biofilm-related infection is now recognized as a common event. The National Institutes of Health revealed that among all microbial and chronic infections, $65 \%$ and $80 \%$, respectively, are associated with biofilm formation [7]. Enhanced survival of exposure to antimicrobials in the biofilm matrix has been demonstrated [8] and biofilm ecology enables gene transfer, quorum sensing and other survivalenhancing activities in response to selective pressures [9]. It is reasonable to suspect that acutely inflamed parenchyma can also support the presence of native biofilms in pneumonia and serious bronchitis.

Herein, we describe the successful isolation of Streptococcus pneumoniae from sputum collected two days after initiation of antipneumococcal therapy using a novel specimen collection method. The study was approved by the study sponsor's institutional review board and informed consent was obtained from the patient prior to specimen collection.
The patient is a 59-year-old woman who was presented to the emergency room in November 2019 with dyspnea. Her past medical history is notable for tobacco use, heavy alcohol use and chronic bronchitis. She had a temperature of $101.2^{\circ} \mathrm{F}$, pulse rate of 123 beats per minute, respiratory rate of 22 breaths per minute and oxygen saturation of $83 \%$ on room air. Chest X-ray demonstrated a left perihilar infiltrate. Physical examination was notable for decreased breath sounds bilaterally, scattered inspiratory rhonchi and tachycardia. Leukocyte count was 17.1 with $89 \%$ neutrophils. Urine Pneumococcal antigen was positive. Urine Legionella antigen was negative. Influenza A/B PCR was negative. She was started empirically on ceftriaxone and azithromycin. Blood cultures were negative. Sputum culture was not collected until the second day of admission as she was unable to expectorate.

\section{Methods}

Approximately two milliliters of expectorated sputum were mixed thoroughly using a sterile swab and was then divided into two equal amounts. This was performed within 3 hours of expectoration. The first was sent to the microbiology laboratory in standard fashion (sterile plastic culture cup) and the second partition was sent in a novel transport/homogenization device. The proprietary device contains a fenestrated internal screen for mechanical homogenization, a chemical biofilm disruption component, and a non-animal based nutritive broth.

${ }^{*}$ Correspondence to: Thomas A Cumbo, Infectious Diseases Division and CMO, Mount St. Mary's Hospital, Catholic Health of Buffalo, Buffalo, NY, USA, E-mail: Thomas.cumbo@chsbuffalo.org

Key words: Streptococcus pneumoniae, sputum, anti-pneumococcal therapy, biofilm disruption technique

Received: October 27, 2019; Accepted: November 19, 2020; Published: November 26, 2020 
After placing the sputum into the device, it was agitated by hand for approximately five seconds. Both samples were sent to our off-site micro lab using a standard courier. Upon arrival in the lab, both samples were plated onto blood, chocolate and MacConkey's agar using standard swab technique. They were incubated in $95 \% \mathrm{Co}_{2}$ at $37^{\circ} \mathrm{C}$ for 48 hours and were then examined for growth.

\section{Results}

The sample transported in the novel transport medium grew few colonies of $S$. pneumoniae and moderate colonies of commensal organisms while the control sample grew only moderate colonies of commensal organisms (Figures 1-3). The Pneumococcus isolated was sensitive to ceftriaxone and resistant to azithromycin in vitro.

\section{Discussion}

We hypothesize that this technique disrupts biofilms present in sputum and exposes sessile bacteria to a nutritive broth allowing propagation of planktonic bacteria. These bacteria appear to survive antimicrobial pressure while protected in biofilms.

The technique employs three putative mechanisms including homogenization of the sample, chemical and mechanical biofilm disruption, and placement into a nutritive broth at the time of collection.

Homogenization of the sample ensures that pathogens are evenly dispersed. Prior work has demonstrated marked disparity of pathogen distribution and concentration in native sputum [10]. Standard loop or swab sampling of native sputum may miss the pathogen entirely.

Bacterial biofilms are inherently resistant to antimicrobial agents [11]. Once bacteria are released from the complicated matrix of the biofilm, however, it is reasonable to conclude that some will revert
A

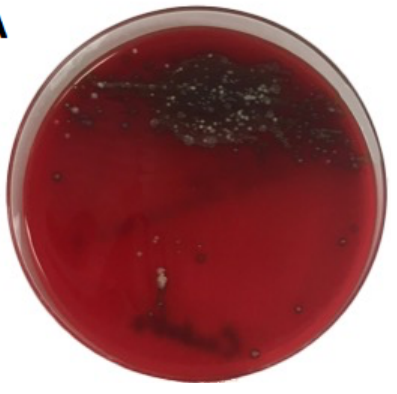

B

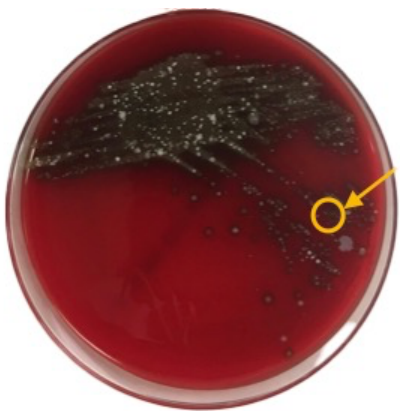

Figure 1. (A) Control sample with moderate colonies of commensal organisms (B) Novel transport medium that grew few colonies of $S$. pneumoniae and moderate colonies of commensal organisms

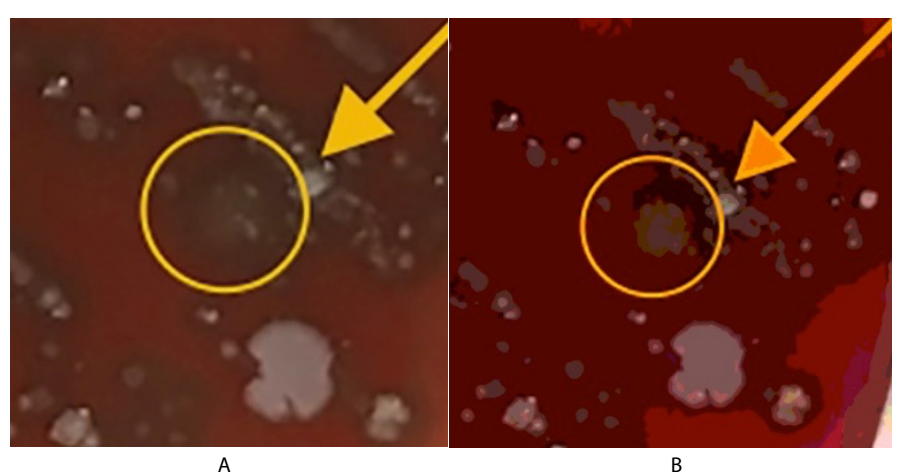

Figure 2. Mucoid colony (A) close up (B) close up - posterized. Circled area represents a large alpha-hemolytic mucoid colony only present on control sample

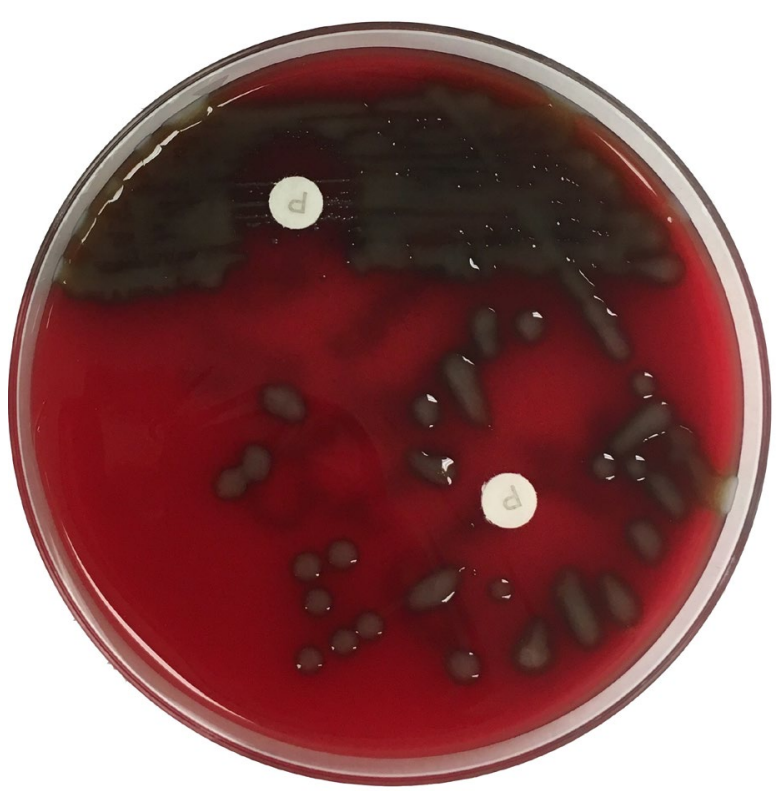

Figure 3. Subculture of the mucoid colony demonstrating Opticin Sensitivity

back to planktonic (and therefore culturable) forms as opposed to the relatively inert and antibiotic impervious sessile forms. This appears likely in our patient since the isolate was proven sensitive to ceftriaxone therapy.

Of note, the patient demonstrated S. pneumoniae with macrolide resistance. We were able to deescalate therapy by stopping empirical atypical coverage and transitioning to oral cefpodoxime. The presence of macrolide resistance was important to note. It is imperative that these data are recognized for both patient care, epidemiological surveillance and hospital antibiograms.

In summary, a combination technique of homogenization, biofilm disruption and nutritive broth facilitates the diagnosis of common pathogens not found using standard techniques and has clinical implications. Given current limitations of sputum culture methodologies that have a low diagnostic yield together with antimicrobial resistance in biofilm formation, this novel technique holds promise in isolation and in combination with molecular methods. Future studies will include studies of unrelated bacteria.

\section{References}

1. Jefferson H, Dalton HP, Escobar MR, Allison MJ (1975) Transportation Delay and the Microbiological Quality of Clinical Specimens. Am J Clin Pathol 64: 689-693. [Crossref]

2. Yourassowsky E (1980) Collection and Transport of Specimens for Bateriological Analysis A Neglected Subject in Medical Teaching. Infection Suppl 2: S143-S145. [Crossref]

3. May JR, Delves DM (1964) The Survival of Haemophilus influenzae and Pneumococci in Specimens of Sputum Sent to the Laboratory by Post. J Clin Pathol 17: 254-256. [Crossref]

4. Tilton RC, Maderaza E, Iannini P, Quintiliani R (1974) The Bacteriological Examination of Sputum. Ann Clin Lab Sci 4: 60-63. [Crossref]

5. Muraki M, Kitaguche S, Ichihashi H, Tsuji F, Ohmori T, et al. (2006) Use of Transport Medium in Sputum Bacterial Culture Examination of Lower Airway Infection. Nihon Kokyuki Gakkai Zasshi 44: 425-430. [Crossref]

6. Metlay JP, Waterer GW, Long AC, Anzueto A, Brozek J, et al. (2019) Diagnosis and Treatment of Adults with Community-acquired Pneumonia. An Official Clinical Practice Guideline of the American Thoracic Society and Infectious Diseases Society of America. Am J Respir Crit Care Med 200: e45-e67. [Crossref]

7. Jamal M, Ahmad W, Andleeb S, Jalil F, Imran M, et al. (2018) Bacterial Biofilm and Associated Infections. J Chin Med Assoc 81: 7-11. [Crossref] 
Cumbo TA (2020) Successful culture of Streptococcus pneumoniae from sputum despite dual anti-pneumococcal therapy using a novel biofilm disruption technique

8. Flemming HC, Wingender J, Szewzyk U, Steinberg P, Rice S, et al. (2016) Biofilms: an Emergent Form of Bacterial Life. Nat Rev Microbiol 14: 563-575. [Crossref]

9. Donlan RM (2002) Biofilms: Microbial Life on Surfaces. Emerg Infect Dis 8: 881-889. [Crossref]
10. May JR (1953) The Bacteriology of Chronic Bronchitis. Lancet 265: 534-537. [Crossref]

11. Costerton JW, Stewart PS, Greenberg EP (1999) Bacterial Biofilms: A common Cause of Persistent Infections. Science 284: 1318-1322. [Crossref]

Copyright: (C2020 Cumbo TA. This is an open-access article distributed under the terms of the Creative Commons Attribution License, which permits unrestricted use, distribution, and reproduction in any medium, provided the original author and source are credited. 